\title{
From discrete multi-exponential model to lifetime distribution model and power law fluorescence decay function
}

\author{
Borys Kierdaszuk \\ Department of Biophysics, Institute of Experimental Physics, University of Warsaw, \\ 93 Zwirki $i$ Wigury St., 02-089 Warsaw, Poland \\ Tel.: +48 22 5540784; Fax: +48 22 5540771; E-mail: borys@biogeo.uw.edu.pl
}

\begin{abstract}
Experimental and theoretical studies of the fluorescence intensity decays in biomolecular systems showed that under constraints of typical experiment fluorescence lifetime distribution is given by gamma function, which led to a power-like decay function. The latter well fits complex (heterogeneous) as well as simple mono-exponential decays. Fluorescence decay kinetics is described by mean value of lifetime distribution characterizing the average rate of the excited-state decay, and one new parameter of heterogeneity $(1<q<3 / 2)$ describing the relative variance of distribution, and objectively reflecting physical heterogeneity of the system. In the classical limit, when $q \rightarrow 1$, the gamma distribution becomes the Dirac delta function, and decay function converges from power-like form to the single-exponential form. Numerous examples illustrate successful applications of this model to rational analysis of complex fluorescence decays of biomacromolecules, e.g., complexes of $E$. coli purine nucleoside phosphorylase (PNP-I) with formycin A (FA, inhibitor) and orthophosphate $\left(P_{i}\right.$, cosubstrate), which led to identification of the mechanism of deactivation of excited state and the $\mathrm{N}(2) \mathrm{H}$ tautomeric form of FA selectively bound by PNP-I. The latter is of great importance for the studies of the mechanism of protein (enzyme) action as well as for more rational drug design.
\end{abstract}

Keywords: Emission spectroscopy, fluorescence intensity decay, multi-exponential model, lifetime distribution, power-like function, enzyme-ligand binding
Abbreviations
FA formycin A;
$P_{i} \quad$ orthophosphate;
PNP-I E. coli purine nucleoside phosphorylase (the product of the deoD gene).

\section{Introduction}

In general the excited state of a fluorophore is sensitive to the physical and chemical nature of its environment, which is a major reason why fluorescence techniques are so frequently used to study dynamics of biological molecules as well as excited state processes, but sensitivity makes the time-resolved fluorescence signal very complex for interpretation. According to traditional multi-exponential model the emission of each photon is considered as a random event, and each fluorophore has the same probability of emitting in a given period of time. It is based on a widely assumed and accepted concept of discrete 
components in fluorescence decays symbolized by multi-exponential functions, i.e. sum of discrete exponential terms described by amplitudes $\left(\alpha_{0 i}\right)$ and lifetimes $\left(\tau_{i}\right)$, which characterize non-interacting resonance transitions. Such a model is often arbitrary, and it is tactically assumed, and widely believed that introduction of additional exponential term (two extra parameters), although without physical significance is justified unless improves fittings. Usually there is lack of molecular model manifested by no correlation between number of exponential terms and biomolecule (protein) conformation [9], e.g., with population of rotamers in the ground state of tryptophan [14] or tyrosine residues [13]. Even for the indole moiety in solution, which exhibited two perpendicular absorption transitions $\left({ }^{1} L_{a},{ }^{1} L_{b}\right)$ to a slightly degenerated state, and one emission transition $\left({ }^{1} L_{a}\right)$, it is difficult to justify two exponential components ( $\tau \sim 0.6$ and $\sim 3 \mathrm{~ns}$ ) in fluorescence decay [2].

Very high sensitivity to physical and chemical environment makes the fluorescence signal too complex to be easy interpreted. Decays of fluorescence intensity in biological systems (e.g., proteins, nucleic acids) often exhibit an extremely complex pattern, even in the case of biomacromolecules containing single fluorescent probe. The origin of complexity can result from (i) multitude conformational substates in the ground and excited states with possible time-dependent interconversions between them in the time scales ranging from femtoseconds to nanoseconds [3], (ii) dynamics, including fluctuations in the ps-ns time scale, (iii) excited-state reactions, e.g., charge (proton, electron) or energy transfer, and troubles with identification of potential acceptors, (iv) relaxations, and (v) interactions with heterogeneous (dipolar) environment [10]. Consequently, there is difficult to find unique conformations or excited-state processes corresponding to discrete decay components. Biomacromolecules with many fluorophores, e.g., proteins containing several tryptophan and/or tyrosine residues, are even much more complicated. In general, excited state decay of the natural or synthetic fluorescence probes in biological systems should reflect such dynamical picture, because particular photons may be emitted from not fully relaxed states.

\section{Materials and methods}

\subsection{Materials}

Purine nucleoside phosphorylase (PNP-I) from E. coli (the product of the $d e o D$ gene) was purified to apparent homogeneity as described earlier [5]. Formycin A (FA), $N$-2-hydroxyethylpiperazine- $N^{\prime}-2$ ethanesulfonic acid (HEPES), mono- and dibasic sodium phosphates were products of Sigma Chemical Co. (USA). Concentrations of FA were determined spectrophotometrically on a Varian Cary-50 instrument (Australia) at $\mathrm{pH} 7$, using $\lambda_{\max }=294 \mathrm{~nm}\left(\varepsilon=10.3 \times 10^{3} \mathrm{M}^{-1} \mathrm{~cm}^{-1}\right)$.

\subsection{Time-resolved fluorescence measurements}

Fluorescence intensity decays of E. coli PNP-I (tyrosine protein), FA and their ternary complex (PNPFA $-P_{i}$ ) for $35 \mu \mathrm{M}$ PNP and $35 \mu \mathrm{M}$ FA in $50 \mathrm{mM}$ Hepes (pH 7) in the presence of $10 \mathrm{mM}$ phosphate $\left(P_{i}\right)$ buffer, at $25^{\circ} \mathrm{C}$ were obtained by time-correlated single photon-counting measurements, as described previously [7]. Typical fluorescence intensity decay data were analyzed by the Marquardt-Levenberg method of nonlinear fitting of one-, two- or three-exponential function, and power-like function (Eq. (4)), each convoluted interactively with the instrument response function (IRF) using homemade procedure under Matlab. The quality of fits was evaluated by the reduced chi-square values, and the structure observed in the plots of residuals normalized to error, i.e. residuals $=\left(I_{\exp }-I_{\text {theo }}\right) / \sqrt{I_{\exp }+I_{\text {theo }}}$, where 
$I_{\text {exp }}$ is the number of experimental counts, and $I_{\text {theo }}$ is the number of counts resulted from convolution of the fitted function with IRF. Thus the $I_{\text {theo }}$ is also subjected to experimental error, and, therefore, must be taken into account in normalization of residuals.

\subsection{Gamma distribution of excited state lifetimes and power-like decay function}

We present here an alternative model based on the idea of distribution of excited state lifetimes, where decay function is given by sum of the individual decays weighted by the amplitudes $P(\tau)$, which better describes the contribution of each component to the fluorescence decay.

$$
I(t)=\int_{0}^{\infty} P(\tau) \exp \left(-\frac{t}{\tau}\right) \mathrm{d} \tau .
$$

It links the character of the decay law to the distribution of lifetimes, more relevant than a sum of discrete exponential terms

$$
I(t)=\sum_{i=1}^{M} \alpha_{0 i} \exp \left(-t / \tau_{i}\right)
$$

characterized by amplitudes $\left(\alpha_{0 i}\right)$ and lifetimes $\left(\tau_{i}\right)$. Lifetime distributions were used so far without a theoretical basis, because distribution functions of pre-exponential factors $\alpha_{i}(\tau)$ were arbitrarily selected as Gaussian or Lorentzian. In this approach a part of the distribution exists for $\tau<0$ (cut-off problem), and, therefore, needs additional normalization [10].

The appropriate distribution $P(\tau)$ under certain experimental constraints was found using maximum entropy method [11], which led to the distribution function $P(\gamma)$ of fluorescence decay rates $(\gamma=1 / \tau)$ that maximizes the information entropy $H=-\int P(\gamma) \ln P(\gamma) \mathrm{d} \gamma$. The mentioned constraints are on the normalization $\left(\int P(\gamma) \mathrm{d} \gamma=1\right)$, the mean value of the decay rate specified as $\langle\gamma\rangle=1 / \tau_{0}$, and the mean value of the logarithm $\langle\ln (\gamma)\rangle$. The latter expresses the fact that distribution is determined only for $\gamma>0$. The distribution $P(\gamma)$, which maximizes the entropy function with the above constraints is given in the form of the gamma distribution

$$
P_{q}(\gamma) \mathrm{d} \gamma=\frac{1}{\Gamma(1 /(q-1))}\left(\frac{1}{(q-1)\langle\gamma\rangle}\right)\left(\frac{\gamma}{(q-1)\langle\gamma\rangle}\right)^{(2-q) /(q-1)} \exp \left(\frac{\gamma}{(q-1)\langle\gamma\rangle}\right),
$$

where $q$ is the parameter of heterogeneity describing the number of decay channels $(N)$ and relative variance $(\omega)$ of fluctuations of $\gamma=1 / \tau$ around the $\langle\gamma\rangle=1 / \tau_{0}$ value

$$
q=1+\frac{2}{N}=1+\frac{\left\langle(\gamma-\langle\gamma\rangle)^{2}\right\rangle}{\langle\gamma\rangle^{2}}=1+\omega
$$

Introduction of the distribution $P(\gamma)$ in the gamma form into kinetic equation (Eq. (1)), leads to decay function

$$
I(t)=\frac{2-q}{\tau_{0}}\left[1-(1-q) \frac{t}{\tau_{0}}\right]^{1 /(1-q)}
$$


described by mean value of lifetime distribution $\left(\tau_{0}\right)$ and parameter of heterogeneity $(q)$. The factor $(2-q) / \tau_{0}$ in the power-like decay function results from normalization. The mean decay time $\left\langle t_{p}\right\rangle$ is given by $\left\langle t_{p}\right\rangle=\tau_{0} /(3-2 q)$. Taking into account decay with $N$ decay channels, the total decay rate $\gamma$ is expected to be a sum of a number $N$ of partial rates $\gamma_{i}$

$$
\gamma=\sum_{1=1}^{N} \gamma_{i} .
$$

In that case, $q=2 / N+1$, i.e. $N=2 /(q-1)$, and gamma function is in the form as follows

$$
P_{N}(\gamma) \mathrm{d} \gamma=\frac{1}{\Gamma(N / 2)}\left(\frac{N}{2\langle\gamma\rangle}\right)\left(\frac{N \gamma}{2\langle\gamma\rangle}\right)^{N / 2-1} \exp \left(-\frac{N \gamma}{2\langle\gamma\rangle}\right)
$$

It is worth noticing that the normalization of power-like decay function leads to constraint on the $q$ values $(q<2)$, which, through $N=2 /(q-1)$ implies constraint on $N(N>2)$. Furthermore, requirement of existence of the mean value of decay time $\left\langle t_{p}\right\rangle=\tau_{0} /(3-2 q)$ implies that $q<3 / 2$, hence $N>4$. Figure 1 shows the gamma distribution (Eqs (2) and (5)) for several values of $q$ and $N$, including the fact that in the classical limit, when the number of decay channels goes to infinity, i.e. $q \rightarrow 1$, the gamma distribution becomes the Dirac delta function, and decay function converges from power-like form to the single-exponential form. When the heterogeneity parameter $(q)$ value increases stepwise from 1 to $3 / 2$, deviation from single-exponential form increases, and it is more pronounced for the tail part of each decay. Concomitantly, this is reflected in an increasing number of exponential components in multiexponential function needed for satisfactory fits (see also Fig. 2 and Table 1). On the other hand, in the limit of a single decay channel, i.e. in the case with one non-interacting resonance transition, there is no distribution of decay rates and, consequently, the fluorescence decay can be described by power-law decay function with $N=1$, so-called compressed hyperbola, widely applied for interpretation of the excited state decays [1].

\section{Results and discussion}

Applicability of gamma distribution model and power law decay function was confirmed so far for interpretation of non-exponential fluorescence decays of tryptophan and tyrosine residues [16,18-20]: (i) their zwitterionic forms and $\mathrm{N}$-acetyl amide analogues in aqueous solutions; (ii) tryptophan residue in two-tryptophan protein (horse liver alcohol dehydrogenase, LADH); and (iii) tyrosine residues in E. coli purine nucleoside phosphorylase (PNP-I) containing 6 tyrosine residues per subunit, and no tryptophan. The latter was now extended to interpretation of fluorescence intensity decays of PNP-I, C-nucleoside formycin A (FA), structural analogue of adenosine (a natural substrate of the E. coli PNP-I), in their ternary PNP-FA- $P_{i}$ complexes in the presence of orthophosphate $\left(P_{i}\right.$, a co-substrate), and compared with previously described results of multi-exponential model [6].

\subsection{Interpretation of fluorescence intensity decays of the enzyme-ligand complexes by power law model}

Purine nucleoside phosphorylases (PNPs) are ubiquitous enzymes which catalyze the reversible cleavage, by phosphorolysis, of the $\mathrm{N}$-glycosidic bond of $\beta$-D-(deoxy)ribonucleosides to yield $\alpha$-D(deoxy)ribose-1-phosphate and the free purine base [12]. Mammalian PNPs and some bacterial enzymes, 


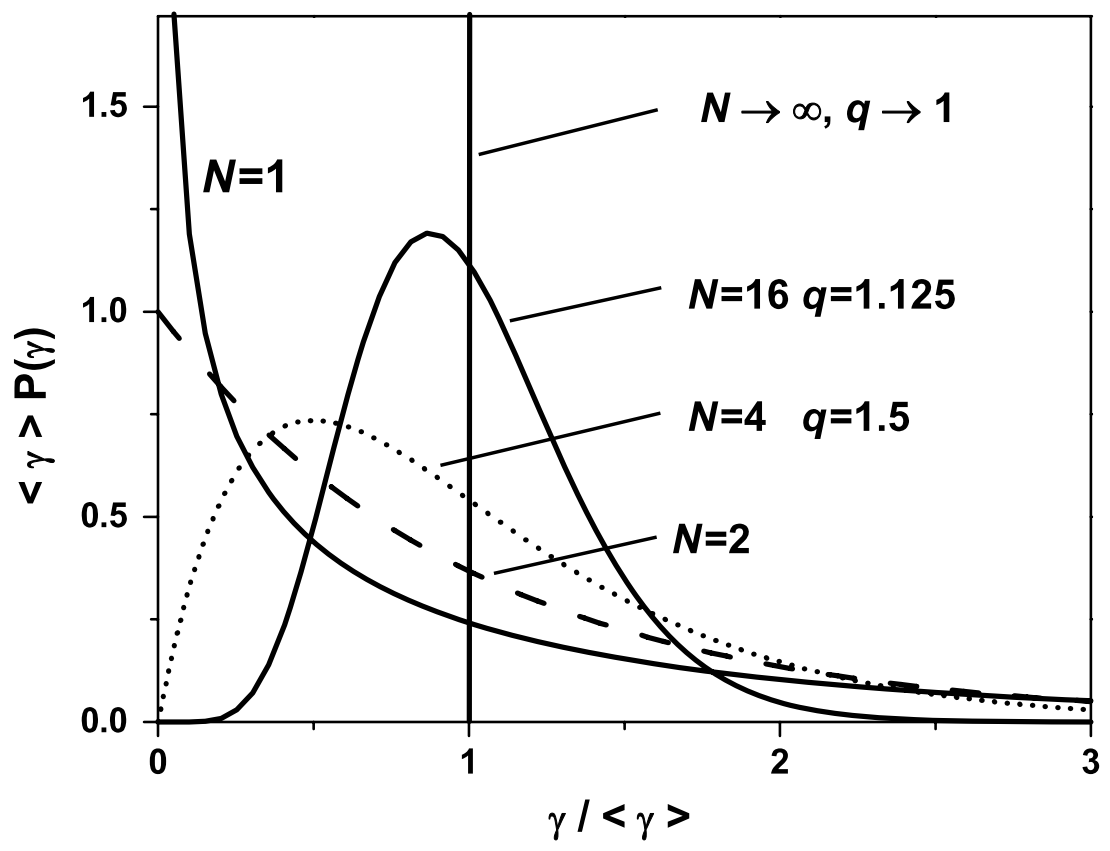

Fig. 1. Gamma distribution function at $N=1,2,4(q=1.5), 16(q=1.125)$ and $N \rightarrow \infty(q \rightarrow 1)$. The latter indicate the Dirac delta function.

e.g., E. coli PNP-II, the product of the xapA gene, are specific for 6-oxopurine nucleosides, i.e. guanosine (Guo) and inosine (Ino), while the enzyme from E. coli, the product of the $d e o D$ gene, referred to below as E. coli PNP-I, cleaves adenosine (Ado) more effectively than Ino and Guo.

We would like to emphasize that formycin A (FA) was chosen for binding studies with E. coli PNP-I because of its interesting emission properties [15] as well as biological activity towards studied enzymes [6]. Due to the C-glycosidic bond, FA is resistant for phosphorolytic cleavage by PNPs. In accord with its structural similarity to Ado, FA exhibited selective and relatively strong inhibition of E. coli PNP-I ( $K_{i} \sim 5 \mu \mathrm{M}$ ), and lack of inhibition $v s$ mammalian PNPs. It is known from long ago (see [4] for review) that FA in aqueous medium is an 85:15 equilibrium mixture of the $\mathrm{N}(1)-\mathrm{H}$ and $\mathrm{N}(2)-\mathrm{H}$ prototropic tautomers. Tautomeric forms of FA differ in both absorption and emission (fluorescence and phosphorescence) properties $[4,6,15,17,21]$, which enable their identification [4,21]. Absorption and emission spectra of the $\mathrm{N}(2)-\mathrm{H}$ form are shifted to the reed by $\approx 5 \mathrm{~nm}$, hence it may be selectively excited at $315 \mathrm{~nm}$ and its fluorescence and phosphorescence emission may be observed at about 365 and $540 \mathrm{~nm}$, respectively. Fluorescence emission of the N(2)-H form of FA in the complex with PNP-I is enhanced by fluorescence resonance energy transfer (FRET) from Tyr160 of the active site and the base moiety of FA $[4,6,21]$. Therefore the studied fluorophore system is very heterogenic, which affects its fluorescence decay.

Absorption and emission properties of FA was used to show that its interaction with PNP-I led to selective binding of the $\mathrm{N}(2)-\mathrm{H}$ form $\left(K_{d}=2.2 \pm 0.2 \mu \mathrm{M}\right)$, and a shift of the tautomeric equilibrium in favour of the latter, independently of $P_{i}$ [6]. Now it is also confirmed by studies on time-resolved fluorescence of PNP-FA- $P_{i}$ complexes (Table 1). The latter is based on the similar values of $\left\langle t_{p}\right\rangle, q$ and $N$ of FA fluorescence emission in the PNP-FA- $P_{i}$ complex $\left(\left\langle t_{p}\right\rangle=1.49 \pm 0.04 \mathrm{~ns}, q=0.105 \pm 0.006\right.$ and $N \sim 19)$ and that determined for the $\mathrm{N}(2)-\mathrm{H}$ tautomeric form of FA $\left(\left\langle t_{p}\right\rangle=1.53 \pm 0.04 \mathrm{~ns}\right.$, 

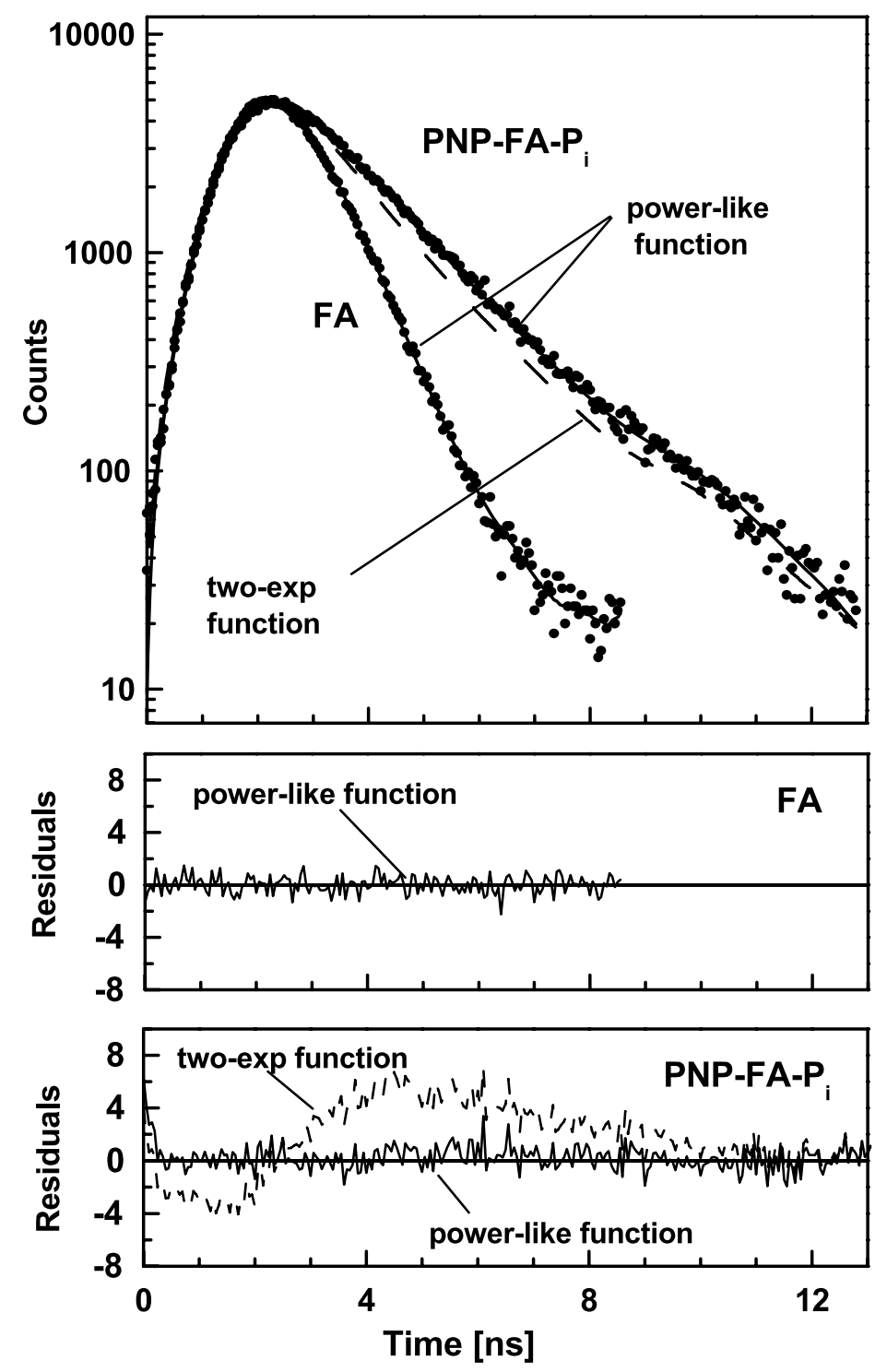

Fig. 2. Fluorescence intensity decays $\left(\lambda_{\mathrm{exc}} / \lambda_{\mathrm{em}}=270 / 335 \mathrm{~nm}\right)$ of FA and its ternary complex with E. coli PNP-I and $P_{i}$ $\left(\right.$ PNP-FA- $\left.P_{i}\right)$. The solid and dashed curves represent the theoretical value of the best fits of power-like function (Eq. (4)) and double-exponential function, respectively. The lower panels show normalized residuals (Section 2.2). Note that the best fits of power-like function to fluorescence intensity decays of FA and its ternary complex with PNP-I and $P_{i}$ were obtained with heterogeneity parameter $(q)$ values of $1.01 \pm 0.02$ and $1.105 \pm 0.006$ (Table 1), respectively. Therefore experimental data for FA may be approximated by single-exponential function $\left(\chi_{R}^{2}=0.92\right)$, while this is not possible for the ternary complex, not only by single-exponential function but double-exponential function (solid line) either $\left(\chi_{R}^{2}=1.32\right.$ ).

$q=0.099 \pm 0.006$ and $N \sim 20)$. The latter was selectively detected at $\lambda_{\mathrm{exc}}=315 \mathrm{~nm}$ and $\lambda_{\mathrm{em}}=$ $365 \mathrm{~nm}$, where tyrosine residues of the enzyme do not absorb, and absorption and emission of the N(1)$\mathrm{H}$ tautomer is negligible low [6]. This is, in fact, a good example of identification of the tautomeric forms of protein-bound ligand by time-resolved fluorescence spectroscopy, not distinguishable in X-ray studies [8], where unambiguous determination of protons is limited by the resolution attainable. 
Table 1

Comparison of multi-exponential analysis with power-like analysis of the fluorescence decays of $35 \mu \mathrm{M} E$. coli purine nucleoside phosphorylase (PNP-I), $35 \mu \mathrm{M}$ formycin A (FA) and ternary complex of $35 \mu \mathrm{M}$ E. coli PNP, $35 \mu \mathrm{M}$ FA and $10 \mathrm{mM}$ phosphate (PNP-FA- $\left.P_{i}\right)$ at $25^{\circ} \mathrm{C}$ in $50 \mathrm{mM}$ Hepes buffer $\mathrm{pH} 7.0$; the excitation and emission wavelengths $\left(\lambda_{\mathrm{exc}} / \lambda_{\mathrm{em}}\right)$ were as indicated, and the standard deviations (in brackets) refer to the last digit ${ }^{*}$

\begin{tabular}{|c|c|c|c|c|c|c|c|c|c|}
\hline \multirow[t]{2}{*}{ Compounds } & \multirow[t]{2}{*}{$\lambda_{\mathrm{exc}} / \lambda_{\mathrm{em}}(\mathrm{nm})$} & \multicolumn{3}{|c|}{ Multi-exponential models } & \multicolumn{3}{|c|}{ Power-like model } & \multicolumn{2}{|c|}{$\chi_{R}^{2}$} \\
\hline & & $\alpha_{i}$ & $\begin{array}{c}\tau_{i} \\
(\mathrm{~ns})\end{array}$ & $\begin{array}{c}\left\langle\tau_{\mathrm{e}}\right\rangle^{\#} \\
(\mathrm{~ns})\end{array}$ & $q$ & $\begin{array}{c}\tau_{0} \\
(\mathrm{~ns})\end{array}$ & $\begin{array}{c}\left\langle t_{p}\right\rangle^{\S} \\
(\mathrm{ns})\end{array}$ & $\begin{array}{c}\text { Multi-exp. } \\
\text { models }\end{array}$ & $\begin{array}{c}\text { Power-like } \\
\text { model }\end{array}$ \\
\hline \multirow[t]{2}{*}{ PNP-I } & $270 / 310$ & $0.11(1)$ & $0.66(2)$ & $2.63(8)$ & $1.25(2)$ & $1.65(3)$ & $3.13(5)$ & 1.02 & 1.02 \\
\hline & & $0.89(2)$ & $2.86(1)$ & & & & & & \\
\hline \multirow[t]{3}{*}{ FA } & $270 / 335$ & 1.00 & $0.53(1)$ & $0.53(1)$ & $1.01(2)$ & $0.56(1)$ & $0.57(3)$ & 0.92 & 1.03 \\
\hline & $315 / 365$ & $0.60(3)$ & $0.49(5)$ & $0.70(8)$ & $1.13(2)$ & $0.58(1)$ & $0.78(3)$ & 1.03 & 1.01 \\
\hline & & $0.40(3)$ & $1.02(2)$ & & & & & & \\
\hline \multirow[t]{8}{*}{ PNP-FA- $-P_{i}$} & $270 / 310$ & $0.38(2)$ & $0.52(3)$ & $1.16(7)$ & $1.187(6)$ & $0.87(1)$ & $1.39(3)$ & 1.09 & 1.02 \\
\hline & & $0.62(3)$ & $1.55(1)$ & & & & & & \\
\hline & $270 / 335$ & $0.20(2)$ & $0.49(2)$ & $1.32(8)$ & $1.105(6)$ & $1.18(3)$ & $1.49(4)$ & 1.32 & 1.07 \\
\hline & & $0.80(3)$ & $1.52(2)$ & & & & & & \\
\hline & $270 / 365$ & $0.25(2)$ & $0.66(7)$ & $1.37(6)$ & $1.099(6)$ & $1.23(2)$ & $1.53(3)$ & 1.07 & 1.04 \\
\hline & & $0.75(2)$ & $1.61(1)$ & & & & & & \\
\hline & $315 / 365$ & $0.66(2)$ & $1.15(4)$ & $1.43(9)$ & $1.057(6)$ & $1.38(2)$ & $1.56(3)$ & 1.07 & 1.03 \\
\hline & & $0.34(2)$ & $1.98(4)$ & & & & & & \\
\hline
\end{tabular}

Notes: *Parameter of heterogeneity $(q)$ and the mean value of lifetime distribution $\left(\tau_{0}\right)$ were obtained from fittings of power-like decay function (Eq. (4)) as described in Section 2.2; ${ }^{\#}\left\langle\tau_{e}\right\rangle=\sum_{i} \alpha_{i} \tau_{i} ;{ }^{\S}\left\langle t_{p}\right\rangle=\frac{\tau_{0}}{3-2 q}$.

The same set of fluorescence decay data of PNP-FA- $P_{i}$ complex was also analyzed using one- and two-exponential function with pre-exponential factors (alphas) given by the Gaussian or Lorentzian distribution of lifetime values of components. As judged on the values of reduced chi-square $\left(\chi_{R}^{2}\right)$ and residuals, one-exponential function was not acceptable $\left(\chi_{R}^{2}=2.5\right)$, two-exponential function was better $\left(\chi_{R}^{2}=1.3\right)$, while good fit of three-exponential model was obtained (data not shown), at lack of interpretation of components. In contrast to gamma function (Section 2.3), which is determined only for positive values of the decay rates (Fig. 1), i.e. for $\tau>0$, there is so-called "cut-off problem" with both Gaussian and Lorentzian distribution function, which are also determined for $\tau<0$. These results justify why quality of fitting should not be the only reason for application of specific model.

\subsection{Concluding remarks}

We trust that physical and mathematical justifications of the gamma distribution model of fluorescence lifetimes, and power-like decay function derived from it are adequately clarified (Section 2.3). To our knowledge this approach is superior $v s$. other models. In some cases, where is no straightforward physical (microscopic) interpretation of exponential terms, power law model maybe advantageous to often arbitrarily used multi-exponential models. Particularly it is useful for analysis of fluorescence intensity decays in biological systems, which as such are heterogenic, for example, characterized by 3- or 4-exponential functions providing mathematically satisfactory fits with sets of six or eight parameters (alphas and taus), but without validation. In contrast, fittings of power-like function lead to two physically justified parameters, which objectively characterize complexity of the studied system and provide additional information about its heterogeneity. This is based on the relative variance of fluctuation $(\omega)$ around mean values of lifetime distribution, and the number of decay paths $(N=2 /(q-1))$, 
which can be easily obtained from fitted parameter $q$ (Eq. (3)). With the ternary PNP-FA- $P_{i}$ complex, $q=0.105 \pm 0.006$ (Table 1 ), leading to $\omega=0.105$ and the number of decay paths $(N \approx 19)$, which may be related to numerous phenomena. Some of them should be considered as a more important, e.g., shifts in the tautomeric equilibrium of FA in favour of the $\mathrm{N}(2)-\mathrm{H}$ form due to its selective binding to the enzyme, and florescence resonance energy transfer from tyrosine residue(s) to the base moiety of FA in the enzyme-ligand complex.

Results of the fluorescence decay analysis (Fig. 1, Table 1) have unequivocally shown that powerlike function well fits complex (heterogeneous) as well as simple mono-exponential decays. Figure 2 presents examples of the comparison of typical fits of two-exponential function and power-like function to fluorescence intensity decay of the PNP-FA- $P_{i}$ ternary complex. Careful inspection of the plots of residuals normalized to error (Fig. 2) and reduced chi-square values (Table 1) collected from fittings of power-like function $\left(\chi_{R}^{2} \sim 1\right)$ as well as one- and two-exponential functions $\left(\chi_{R}^{2} \gg 1\right)$ confirmed conclusion that fluorescence intensity decays were best fitted by power-like function. Generally nonsatisfactory fits of the double-exponential model were observed with the non-random distribution of residuals (Fig. 2), and elevated values of the reduced chi-square (Table 1). In contrast, improvement of fittings with power-like decay function was obtained with the normalized residual differences randomly distributed around zero (see example in Fig. 2), and reduced chi-square values, which were $\sim 1$ (Table 1).

Results obtained here contribute to our knowledge of the factors involved in the mechanism of enzyme ligand recognition by selective binding of the tautomeric form of ligand. This was performed by time-resolved fluorescence spectroscopy, because the tautomeric forms are not distinguishable in other methods including X-ray studies [8]. An important factor hitherto overlooked, and to which attention has to be draw, relates to the importance of these results for more rational drug design. Accurate determination of the inhibitor structure, acceptable by the enzyme active site and facilitating strong and selective enzyme-inhibitor interaction, is particularly important since PNPs has been considered as a primary target [12] for selective immunosuppressive agents to suppress the host versus graft response in organ transplantation, treatment of T-cell leukaemias, countering T-cell-mediated autoimmune diseases. PNP inhibitors are also the center of interest for hindrance of phosphorolytic cleavage of the antitumour and antiviral purine nucleoside analogues. Differences in specificity between PNPs from E. coli and humans has also been profited from the development of so-called tumour-directed gene therapy.

Application of power law model demonstrate physical attempt of rational analysis of complex fluorescence decay in heterogenic systems of biological macromolecules. This approach is based on the statistical treatment of fluctuations of the characteristic lifetime of fluorescence, and, therefore, generally differ from that presented so far (e.g., multi-exponential model) in terms of physical parameters available. Physical information in the power law function is based on the values of parameter of heterogeneity $(1<q<3 / 2)$ - objectively reflecting physical heterogeneity of the system, and the mean lifetime value from distribution $\left(\tau_{0}\right)$ - characterizing the average rate of the excited-state decay $\left(\langle\gamma\rangle=1 / \tau_{0}\right)$. However, both experimental and theoretical studies of the model compounds are needed to demonstrate how the parameters for this function can be related to the physical characteristics of the system.

\section{Acknowledgement}

This research was supported by the Polish Ministry of Science and Higher Education (Grant No. NN202 105536). 


\section{References}

[1] M.N. Berberan-Santos, E.N. Bodunov and B. Valeur, Mathematical functions for the analysis of luminescence decays with underlying distributions: 2. Becquerel (compressed hyperbola) and related decay functions, Chem. Phys. 317 (2005), 57-62.

[2] P.R. Callis, $1 L_{\mathrm{a}}$ and $1 L_{\mathrm{b}}$ transitions of tryptophan: Applications of theory and experimental observations to fluorescence of proteins, Methods Enzymol. 278 (1997), 113-150.

[3] M. Karplus and A. McCammon, Dynamics of proteins, elements and function, Annu. Rev. Biochem. 53 (1983), $263-300$.

[4] B. Kierdaszuk, Emission spectroscopy of complex formation between Escherichia coli purine nucleoside phosphorylase (PNP) and identified tautomeric species of formycin inhibitors resolves ambiguities found in crystallographic studies, in: Fluorescence Spectroscopy, Imaging and Probes. New Tools in Chemical, Physical and Life Sciences, Vol. 2, R. Kraayenhof, A.J.W.G. Visser and H.C. Gerritsen, eds, Springer-Verlag, Heidelberg, 2002, pp. 277-296.

[5] B. Kierdaszuk, A. Modrak-Wójcik and D. Shugar, Binding of phosphate and sulfate anions by purine nucleoside phosphorylase from E. coli: ligand dependent quenching of enzyme intrinsic fluorescence, Biophys. Chem. 63 (1997), 107-118.

[6] B. Kierdaszuk, A. Modrak-Wójcik, J. Wierzchowski and D. Shugar, Formycin A and its N-methyl analogues, specific inhibitor of E. coli purine nucleoside phosphorylase (PNP): induced tautomeric shifts on binding to enzyme, and enzyme $\rightarrow$ ligand fluorescence resonance energy transfer, Biochim. Biophys. Acta 1476 (2000), 109-128.

[7] B. Kierdaszuk and J. Włodarczyk, Interpretation of intramolecular stacking effect on the fluorescence intensity decay of 3-methylbenzimidazolyl $\left(5^{\prime}-5^{\prime}\right)$ guanosine dinucleotides using a model of lifetime distribution, Eur. Biophys. J. 35 (2006), 424-430.

[8] G. Koellner, M. Luić, D. Shugar, W. Saenger and A. Bzowska, Crystal structure of the ternary complex of E. coli purine nucleoside phosphorylase with formycin B, a structural analogue of the substrate inosine, and phosphate (sulphate) at 2.1 Å resolution, J. Mol. Biol. 280 (1998), 153-166.

[9] A.S. Ladokhin and S.H. White, Alphas and taus of tryptophan fluorescence in membranes, Biophys. J. 81 (2001), 18251827.

[10] J.R. Lakowicz, Principles of Fluorescence Spectroscopy, 3rd edn, Springer-Verlag, Singapore, 2006.

[11] E.W. Montroll and M.F. Schlesinger, Maximum entropy formalism, fractals, scaling phenomena, and $1 / f$ noise: A tale of tails, J. Stat. Phys. 32 (1983), 209-230.

[12] M.J. Pugmire and S.E. Ealick, Structural analyses reveal two distinct families of nucleoside phosphorylases, Biochem J. 361 (2002), 1-25.

[13] J.B.A. Ross, W.R. Laws, K.W. Rousslang and H.R. Wyssbrod, Tyrosine fluorescence and phosphorescence from proteins and polypeptides, in: Topics in Fluorescence Spectroscopy, J.R. Lakowicz, ed., Biochemical Applications, Vol. 3, Plenum Press, New York, 1992, pp. 1-63.

[14] J.B.A. Ross, H.R. Wyssbrod, A. Porter, G.P. Schwartz, C.A. Michaels and W.R. Laws, Correlation of tryptophan intensity decay parameters with ${ }^{1} \mathrm{H}$ NMR-determined rotamer conformations: [Tryptophan ${ }^{2}$ ]Oxytocin, Biochemistry 31 (1992), $1585-1594$.

[15] J. Wierzchowski and D. Shugar, Luminescence studies of formycin, its aglycone, and their N-methyl derivatives: tautomerisation, sites of protonation and phototautomerism, Photochem. Photobiol. 35 (1982), 445-458.

[16] J. Włodarczyk and B. Kierdaszuk, Interpretation of fluorescence decays using a power-like model, Biophys. J. 85 (2003), $589-598$.

[17] J. Włodarczyk and B. Kierdaszuk, Origin of non-exponential fluorescence decays in enzyme-ligand complex, in: Progress in Biomedical Optics and Imaging (Volume 5). Complex Dynamics, Fluctuations, Chaos and Fractals in Biomedical Photonics, V.V. Tuchin ed., Proceedings of SPIE, Vol. 5330, SPIE, Bellingham, USA, 2004, pp. 92-100.

[18] J. Włodarczyk and B. Kierdaszuk, A new approach to interpretation of heterogeneity of fluorescence decay: effect of induced tautomeric shift and enzyme $\rightarrow$ ligand fluorescence resonance energy transfer, Biophys. Chem. 123 (2005), 146153.

[19] J. Włodarczyk and B. Kierdaszuk, Fluorescence decay heterogeneity model based on electron transfer processes in an enzyme-ligand complex, Acta Phys. Polon. 107 (2005), 883-894.

[20] J. Włodarczyk and B. Kierdaszuk, A new approach in interpretation of heterogeneity of fluorescence decay in complex biological systems, in: Diagnostic Optical Spectroscopy in Biomedicine III, M.-A. Mycek, ed., Proceedings of SPIE, Vol. 5862, SPIE, Bellingham, USA, 2005, pp. 205-208.

[21] J. Włodarczyk, G. Stoychev-Galitonov and B. Kierdaszuk, Identification of the tautomeric form of formycin A in its complex with E. coli purine nucleoside phosphorylase based on the effect of enzyme-ligand binding on fluorescence and phosphorescence, Eur. Biophys. J. 33 (2004), 377-385. 


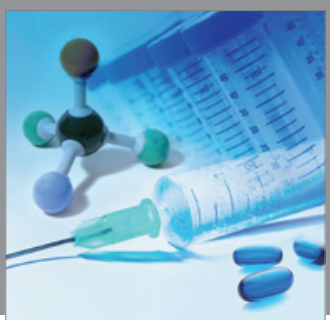

International Journal of

Medicinal Chemistry

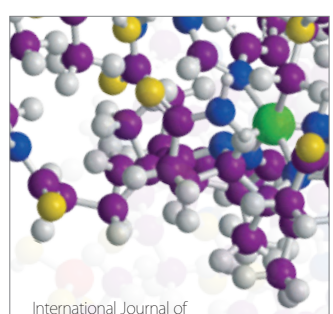

Carbohydrate Chemistry

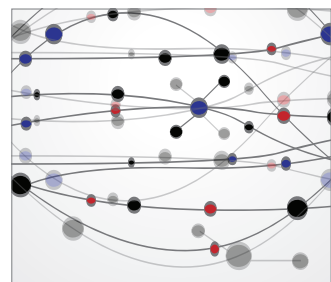

The Scientific World Journal
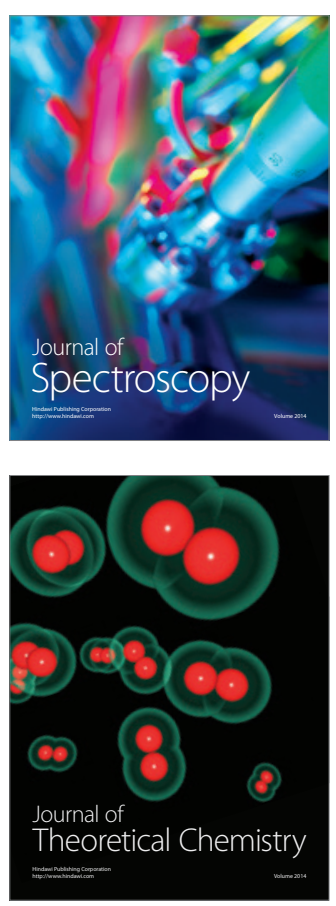
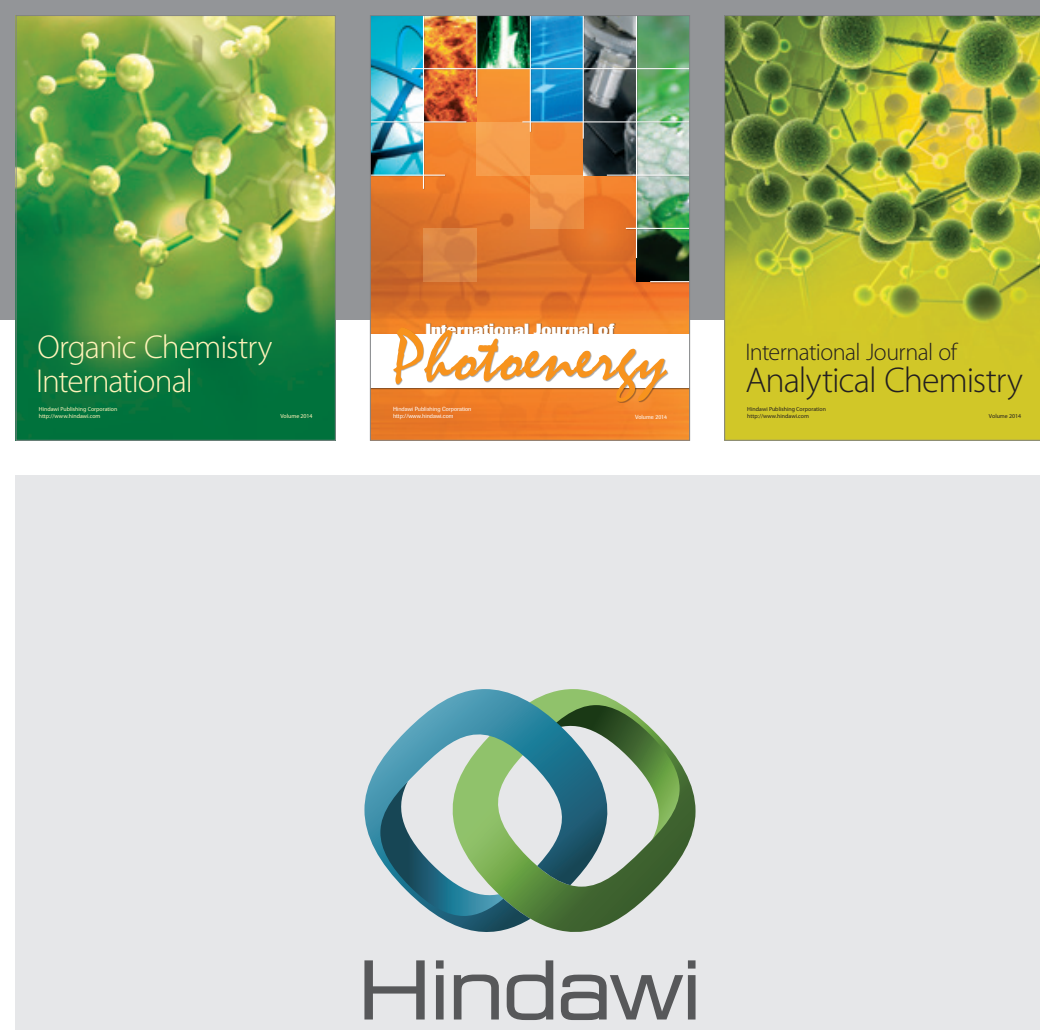

Submit your manuscripts at

http://www.hindawi.com
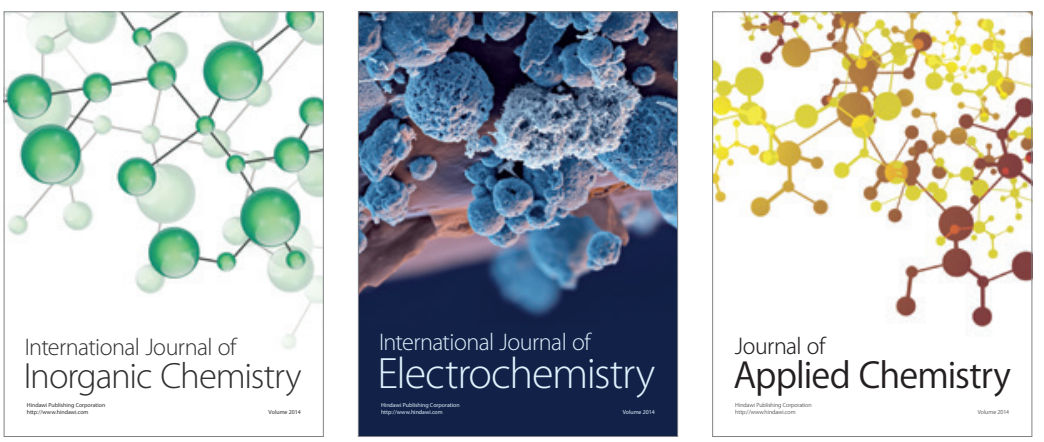

Journal of

Applied Chemistry
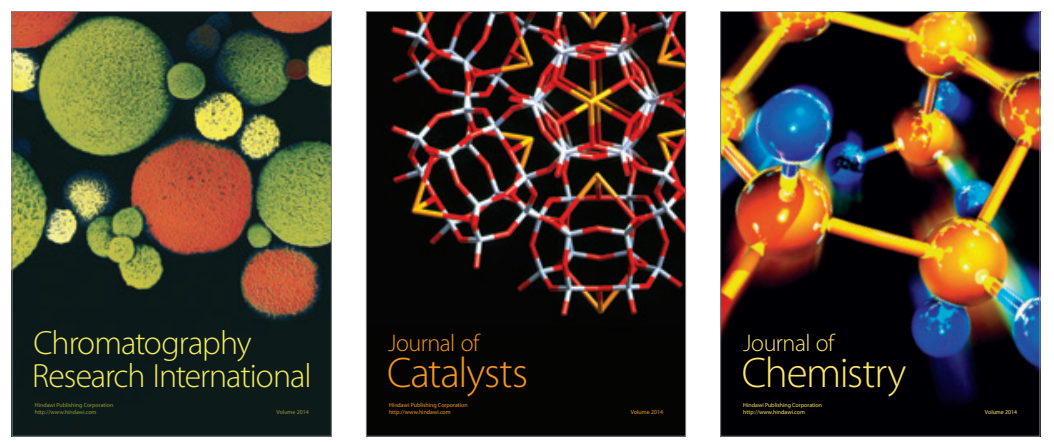
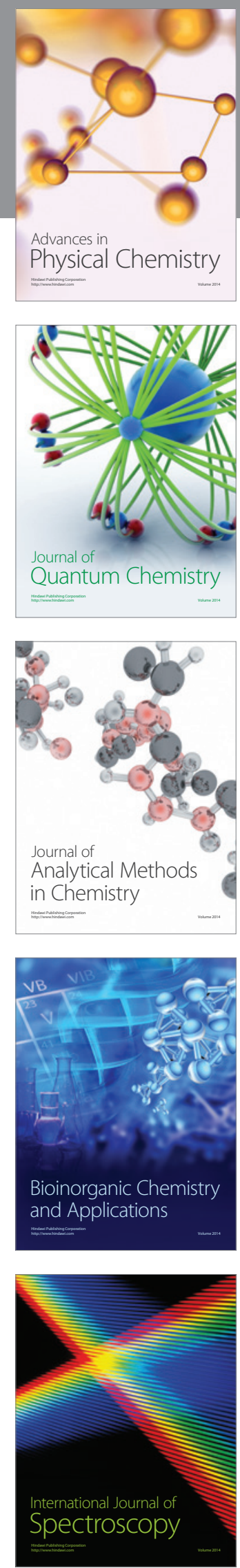